\title{
The Unequal Effects of Social Distancing Policy on Subway Ridership during the COVID-19 Pandemic in Seoul, South Korea
}

\author{
Jaeyoung Ha (D) Suyoung Jo (iD) \\ Hee-kyoung Nam $\cdot$ Sung-il Cho
}

Accepted: 26 October 2021 / Published online: 1 January 2022

(C) The Author(s) 2021

\begin{abstract}
In the Republic of Korea, social distancing policies relied on voluntary participation by citizens and exhibited short-term changes. In this situation, the effects of such policies varied depending on each community's capacity to comply. Here, we collected subway ridership data for 294 stations on nine Seoul Metro lines and aggregated the data for each station to the 184 smallest administrative areas. We found that the mean percent change in subway ridership was fitted by an additive model of the log-transformed percent ratio of the restaurant industry (estimated degrees of freedom $(\mathrm{EDF})=3.24, P<0.001$ ),
\end{abstract}

Supplementary Information The online version contains supplementary material available at https://doi. org/10.1007/s11524-021-00585-4.

J. Ha $\cdot$ S. Jo $\cdot$ H. Nam $\cdot$ S. Cho $(\bowtie)$

Department of Public Health Science, Graduate School of Public Health, Seoul National University, 1 Gwanak-ro, Gwanak-gu, Seoul 08826, Republic of Korea

e-mail: persontime@hotmail.com

J. Ha

e-mail: hajaeyoung92@gmail.com

S. Jo

e-mail: josuyoung1@gmail.com

H. Nam

e-mail: happidemiologist@gmail.com

S. Cho

Institute of Health and Environment, Graduate School of Public Health, Seoul National University, Seoul, South Korea the Deprivation Index (DI) $(\mathrm{EDF}=3.66, P=0.015)$, and the proportion of essential workers $(\beta=-0.10$ (95\% confidence interval -0.15 to $-0.05, P<0.001$ ). We found a distinct decrease in subway ridership only in the least deprived areas, suggesting that social distancing is costly.

\section{Introduction}

Social distancing policy was implemented to prevent the spread of COVID-19 by reducing person-to-person contact [1]. However, studies have discussed the "luxury nature" [2] of social distancing (i.e., unequal impact on human mobility according to socioeconomic status) during the COVID-19 pandemic [3-5]. In the Republic of Korea, social distancing policies strongly relied on the voluntary participation of citizens without mandates [6] and exhibited short-term changes. In this situation, the effects of such policies varied depending on each community's capacity to comply.

Many studies have analyzed human mobility patterns as a proxy for the effects of social distancing policy $[3-5,7]$. Some of them focused on subway ridership [5,8], because daily subway use for transportation is useful for examination of how daily movement patterns change with social distancing policies. Continued subway use during severe COVID-19 waves may indicate groups of people who were unable to work at home or change their transportation mode to a taxi or private car, despite the high-risk perception 
of the subway as a closed, crowded space [9]. The subway ridership pattern can be interpreted from a regional perspective, because it reflects the socioeconomic characteristics of the area around a subway station [10].

Here, we studied the unequal responses of communities to social distancing policy through subway use patterns, which reflected regional socioeconomic characteristics. We hypothesized that areas with higher socioeconomic status would show a greater decrease in subway use. We adjusted for the proportion of essential workers and for the leisure-purpose movements represented by restaurants in areas to explore the relationship.

\section{Methods}

\section{Study Area}

In Seoul, Korea, as of July 2021, there were nine major subway lines with 321 stations and a light line with 10 stations. We studied 294 stations on the nine major subway lines from January 1, 2020, to December 31,2020. We aggregated the card tags of each station to the smallest administrative units of Seoul [11]. We treated stations on different lines differently; we used the mean value of a transfer station with several lines, considering heterogeneity in the amount of movement. Thus, 184 of the 425 administrative areas were included.

\section{Variables}

The response variable was set as the percent change (\%) in the reduction in subway travel between the period with the weaker social distancing level 1 (November 5 to 17, 2020) and the period with the stronger social distancing level 2.5 (December 10 to 22, 2020) (Appendix 1). We compared changes between the two periods to assess community adoption of rapidly strengthened policy. The percent change in subway ridership was averaged weekly to adjust for differences between weekdays and weekends.

The Deprivation Index (DI), density of restaurant industries, and proportion of essential workers were used as explanatory variables. To estimate the socioeconomic status of each region, we used the
DI reported by the Seoul Health Foundation in 2018 [12], which was calculated using the Korean version of the deprivation index (KorDep_2015) developed by Kim et al. [13]. The DI was calculated by summing four standardized scores (z-scores) regarding the following proportions: households with automobiles, the population with less than a high school education, the population corresponding to the lower social class, and the divorced or widowed population.

The percentage of essential workers in each region was calculated as the sum of the numbers of workers engaged in agriculture, forestry, fishing, manufacturing, mining, water, electricity and gas, construction, passenger transportation, wholesale and retail, education, and healthcare, divided by the total number of workers in 2018. The restaurant industry variable was calculated as the log-transformed percentage of restaurant businesses (cafes, restaurants, bakeries, and pubs), compared with the total number of businesses in each region of 2018.

\section{Statistical Analysis}

A generalized additive model (GAM) integrates a generalized linear model and additive model; it flexibly reflects nonlinear associations between response and explanatory variables $[14,15]$. We used a GAM to make inferences concerning the mean percent change of the reduction in subway use (y) caused by the additive effects of socioeconomic factors in each region, and the smooth functions of each variable as:

$$
y=\beta_{0}+\beta_{1} \times \text { essential workers }+\mathrm{s}(\log (\text { restaurants }))+s(\mathrm{DI})+\epsilon
$$

Statistical analyses using GAMs were conducted using the "mgcv" package (ver. 1.8-28) in R ver. 4.0.4. A $p$-value $<0.05$ from a two-sided statistical test was considered statistically significant.

\section{Results}

Figure 1 shows that the subway use decreased during the period when the social distancing policy was strengthened quickly, from November 5, 2020 to December 22, 2020. During this period, subways were used more in deprived areas (Q3, Q4, and Q5) than in less deprived areas (Q1 and Q2). Using the percent change of the reduction (Fig. 1b), we found 
(a)

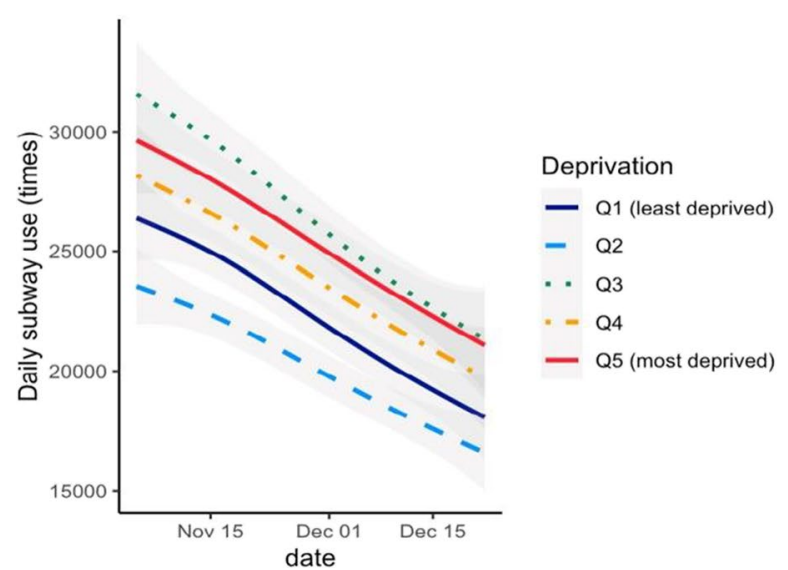

Fig. 1 Changes in subway ridership with socioeconomic deprivation. GAM-smoothed lines with the 95\% confidence intervals of the a weekly averaged subway use and $\mathbf{b}$ percent change in the weekly averaged subway use from November 5

similar percent changes in areas Q3, Q4, and Q5; the reduction in subway use was approximately $30 \%$ greater in the least deprived area $(\mathrm{Q} 1)$. Areas in Q2 had the least subway use, but the reductions in these areas were also smallest during that period.

We constructed a multivariate GAM model that included DI with other socioeconomic covariates (Fig. 2). The log-transformed restaurant industry (Estimated Degrees of Freedom $(\mathrm{EDF})=3.24$, $P<0.001$, Fig. 2a) and DI (EDF $=3.66, P=0.015$, (b)

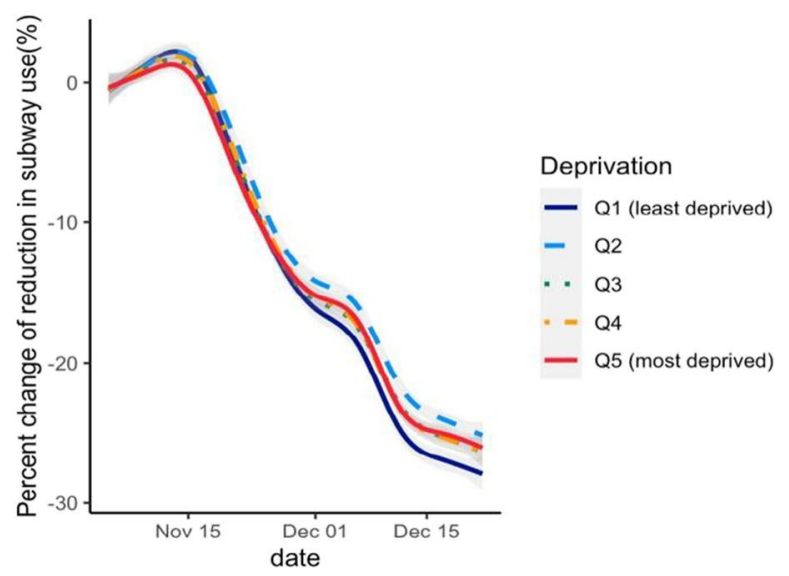

to December 22, 2020, compared with baseline. The DIs of the study areas were classified in quintiles; higher quintiles indicate greater deprivation

Fig. 2b) had significant nonlinear associations with the changes in subway ridership. In comparison, the percentage of essential workers had a linear association with the percent change of subway ridership ( $\beta=-0.10$ (95\% confidence interval -0.15 to -0.05 , $P<0.001$, Fig. 2c). Despite adjustment for the negative effect of essential workers on the percent change of subway ridership, areas where restaurants contributed less than approximately $10 \%$ of businesses were negatively associated with the fitted values. (a)

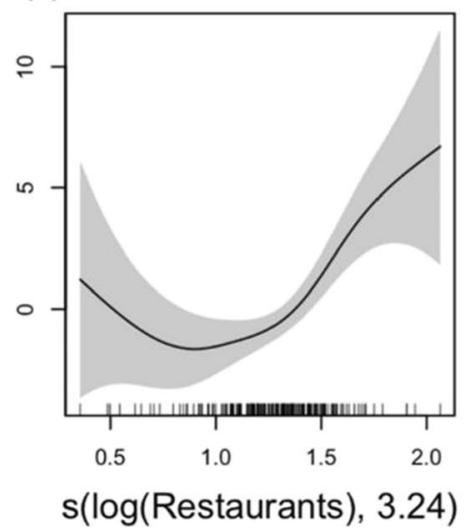

(b)

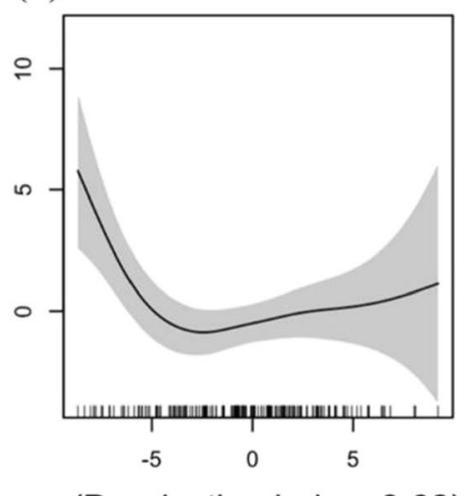

$\mathrm{s}$ (Deprivation index, 3.66) (c)

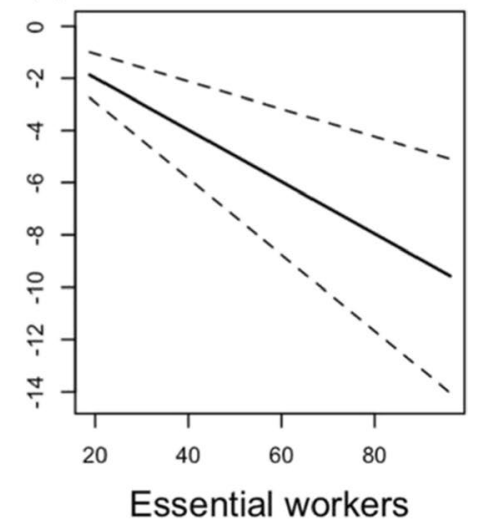

Fig. 2 Effects of socioeconomic socioeconomic characteristic variable on the percent change in the reduction in subway ridership 
Conversely, the percent change in subway ridership reduction was greater in areas where the percent ratio exceeded approximately 30\% (Fig. 2a). The relationship in which less deprived areas had greater percent changes in subway ridership was evident only in areas with DI $<-5$ (Fig. 2b).

\section{Discussion}

This study examined the unequal responses of communities to social distancing policy through differences in the reduction of subway usage by area. Despite adjustment for the effects of essential workers, the DI of a region and density of restaurants significantly contributed to a positive mean percent change in subway ridership. However, these contributions were evident only during a particular interval.

A large percent change in subway ridership was found in areas with a high proportion of restaurants, which indicated that such areas were more commercial in nature. Because highly commercial areas are usually clustered [16], people may avoid visiting such areas because of the risk-perception of crowded places. In comparison, the percent change in subway ridership decreased slightly in areas with a low proportion of restaurants. It is possible that administrative or other essential businesses are present in these areas.

The least deprived areas had the largest percent change in subway ridership. Similar results were observed in studies exploring the association between socioeconomic deprivation and mobility using mobile phone data in Spain and New Zealand. In both studies, mobility levels in low deprivation areas declined strongly during the lockdown period, then normalized more quickly $[3,7]$. Our study is unique in that the association between socioeconomic deprivation and the percent change in subway ridership persisted, despite adjustment for the percentage of essential workers. This suggests that regardless of functional differentiation between essential and non-essential industries in assessed areas, the socioeconomic statuses of the regions caused differences in subway usage patterns.

An important finding was the "threshold effect" in social distancing; a significant reduction in subway ridership was found only in the least deprived areas. This suggests that people below a particular socioeconomic level perceive the costs of social distancing to outweigh their benefits because of economic insecurity [4, 17, 18]. According to Lou et al. [17], additional economic support and employment retention services for vulnerable low-income groups are needed, because structural employment factors may disproportionally affect mobility. The COVID-19 Marmot review [19] also recommended that employment-retention policies, such as the Coronavirus Job Retention Scheme, be extended for low-income workers; subsidies are recommended for firms and dismissed workers as direct and short-term strategies.

Furthermore, there is a need to build a broader, more robust safety net; our findings are consistent with the previous implication that intermediate socioeconomic groups experience a similar economic dilemma when following social distancing policies [19]. The authors of the previous study proposed methods to reduce precarious employment and invest in both quality and active labor market policies [19]. Our results suggest that financial support for more deprived communities is needed to enhance their capacities to provide various social services; support can also enhance the recovery of affected local businesses and employment. The ultimate goals of a community's COVID-19 mitigation strategy, including social distancing, are to enable the community to thrive socially and economically by minimizing the adverse impact of the pandemic, and to end the pandemic by eventually bringing its spread under effective control [20].

Two limitations should be noted when interpreting our results. First, we used aggregated data that cannot explain individual patterns. Second, the age structures of the regions were not considered. Age variations in the proportion of the economically active population may affect mobility patterns. However, regions would have been self-controlled for age differences by determining the percentages between the two periods.

\section{Conclusion}

We found different regional responses to social distancing policies arising from their socioeconomic characteristics. A distinct decrease in subway ridership was found only in the least deprived areas. This suggests the "luxury nature" of social distancing in Seoul. We propose that the economic security of citizens be prioritized to devise equal integrated responses to the epidemic. 
Acknowledgements This research was supported by the Bio \& Medical Technology Development Program of the National Research Foundation (NRF) funded by the Korean government (MSIT) (No. 2021M3E5E3081366). The authors also would like to express their sincere gratitude to the Seoul Open Data Plaza for aggregating the subway ridership data used to conduct this research.

Funding This research was supported by the Bio \& Medical Technology Development Program of the National Research Foundation (NRF) funded by the Korean government (MSIT) (No. 2021M3E5E3081366).

Open Access This article is licensed under a Creative Commons Attribution 4.0 International License, which permits use, sharing, adaptation, distribution and reproduction in any medium or format, as long as you give appropriate credit to the original author(s) and the source, provide a link to the Creative Commons licence, and indicate if changes were made. The images or other third party material in this article are included in the article's Creative Commons licence, unless indicated otherwise in a credit line to the material. If material is not included in the article's Creative Commons licence and your intended use is not permitted by statutory regulation or exceeds the permitted use, you will need to obtain permission directly from the copyright holder. To view a copy of this licence, visit http://creativecommons.org/licenses/by/4.0/.

\section{References}

1. Jawaid A. Protecting older adults during social distancing. Science. 2020;368(6487):145.

2. Huang X, Li Z, Jiang Y, et al. The characteristics of multisource mobility datasets and how they reveal the luxury nature of social distancing in the U.S. during the COVID19 pandemic. Int J Digit Earth. 2021;14(4):424-42. https://doi.org/10.1080/17538947.2021.1886358.

3. Campbell M, Marek L, Wiki J, et al. National movement patterns during the COVID-19 pandemic in New Zealand: the unexplored role of neighbourhood deprivation. $J$ Epidemiol Community Health. 2021;75(9):903. https://doi. org/10.1136/jech-2020-216108.

4. Pinchoff J, Kraus-Perrotta C, Austrian K, et al. Mobility patterns during COVID-19 travel restrictions in Nairobi urban informal settlements: who is leaving home and why. J Urban Health. 2021;98(2):211-21. https://doi.org/10. 1007/s11524-020-00507-w.

5. Sy KTL, Martinez ME, Rader B, White LF. Socioeconomic disparities in subway use and COVID-19 outcomes in New York City. American Journal of Epidemiology. 2020. https://doi.org/10.1093/aje/kwaa277.

6. Hwang YS, Jo HS. Associations between compliance with non-pharmaceutical interventions and social-distancing policies in Korea during the COVID-19 pandemic. J Prev Med Public Health. 2021;54(4):230-7. https://doi.org/10. 3961/jpmph.21.139.

7. Glodeanu A, Gullón P, Bilal U. Social inequalities in mobility during and following the COVID-19 associated lockdown of the Madrid metropolitan area in Spain. Health Place. 2021;70:102580.

8. Park J. Changes in subway ridership in response to COVID-19 in Seoul, South Korea: implications for social distancing. Cureus. 2020;12(4):e7668.

9. Kim M-H, Lee J, Gim T-HT. How did travel mode choices change according to coronavirus disease 2019? Lessons from Seoul, South Korea. Int J Urban Sci. 2021;25(3):43754. https://doi.org/10.1080/12265934.2021.1951823.

10. Lee S, Yi C, Hong S-P. Urban structural hierarchy and the relationship between the ridership of the Seoul Metropoli$\tan$ Subway and the land-use pattern of the station areas. Cities. 2013;35:69-77.

11. Seoul Open Data Plaza. Subway Ridership Data 2020. Seoul: Seoul Metropolitan Government. http://data.seoul.go.kr/ dataList/OA-12252/S/1/datasetView.do. Uploaded Feb 17, 2015. Updated May 5, 2021. Accessed 5 Jan 2021.

12. Wang H, Ham J, Hwang M. 2018 monitoring of health disparities, SEOUL. Seoul: Seoul Health Foundation; 2019. Report No. 51-B553798-000001-10. Available at: https://nl.go.kr. Accessed 5 Jan 2021.

13. Kim D, Lee S, Ki M, et al. Developing health inequalities indicators and monitoring the status of health inequalities in Korea. Sejong: Korea Institute for Health and Social Affairs; 2013. https://www.kihasa.re.kr/publish/report/ view?type $=$ all\&seq=27544. Accessed 5 Jan 2021.

14. Hastie T, Tibshirani R. Generalized additive models. Stat Sci. 1986;1(3):297-310. https://doi.org/10.1214/ss/1177013604.

15. Dominici F, McDermott A, Zeger SL, Samet JM. On the use of generalized additive models in time-series studies of air pollution and health. Am J Epidemiol. 2002;156(3):193-203. https://doi.org/10.1093/aje/kwf062.

16. Moss R, Naghizade E, Tomko M, Geard N. What can urban mobility data reveal about the spatial distribution of infection in a single city? BMC Public Health. 2019;19(1):1-16.

17. Lou J, Shen X, Niemeier D. Are stay-at-home orders more difficult to follow for low-income groups? J Transp Geogr. 2020;89:102894. https://doi.org/10.1016/j.jtrangeo.2020.102894.

18. Choi H, Kim S-Y, Kim J-W, et al. Mainstreaming of health equity in infectious disease control policy during the COVID-19 pandemic era. J Prev Med Public Health. 2021;54(1):1.

19. Marmot M, Allen J, Goldblatt P, Herd E, Morrison J. Build back fairer: the COVID-19 Marmot review. The Pandemic, Socioeconomic and Health Inequalities in England. London: Institute of Health Equity; 2020. https:// www.health.org.uk/publications/build-back-fairer-thecovid-19-marmot-review. Accessed 5 Oct 2021.

20. Centers for Disease Control and Prevention. An approach for monitoring and evaluating community mitigation strategies for COVID-19. Updated November 13, 2020. https://www.cdc.gov/coronavirus/2019-ncov/php/monit oring-evaluating-community-mitigation-strategies.html. Accessed 21 Jul 2021.

Publisher's Note Springer Nature remains neutral with regard to jurisdictional claims in published maps and institutional affiliations. 